\title{
Psychometric properties of the Portuguese version of the Yale Food Addiction Scale
}

\author{
Sandra Torres ${ }^{1,2}$ - Marta Camacho ${ }^{3}$ Patrício Costa ${ }^{4,5,1} \cdot$ Gabriela Ribeiro $^{3,6}$. \\ Osvaldo Santos $^{7,8} \cdot$ Filipa Mucha Vieira $^{1}$ - Isabel Brandão, ${ }^{9,10}$ - Daniel Sampaioo ${ }^{11,12}$. \\ Albino J. Oliveira-Maia ${ }^{3,13,14,15}$
}

Received: 7 May 2016/Accepted: 15 December 2016/Published online: 18 January 2017

(C) Springer International Publishing Switzerland 2017

\begin{abstract}
Purpose Rising rates of obesity have been recently associated to the novel concept of food addiction (FA). The Yale Food Addiction Scale (YFAS) is the most widely used measure for examining FA (1) and analysis of its reliability and validity is expected to facilitate empirical research on the construct. Here, we tested the psychometric properties of a Portuguese version of the YFAS (P-YFAS), establishing its factor structure, reliability and construct validity. Methods Data were obtained from 468 Portuguese individuals, 278 sampled from non-clinical populations, and
\end{abstract}

This article is part of the topical collection on Food addiction.

Electronic supplementary material The online version of this article (doi:10.1007/s40519-016-0349-6) contains supplementary material, which is available to authorized users.

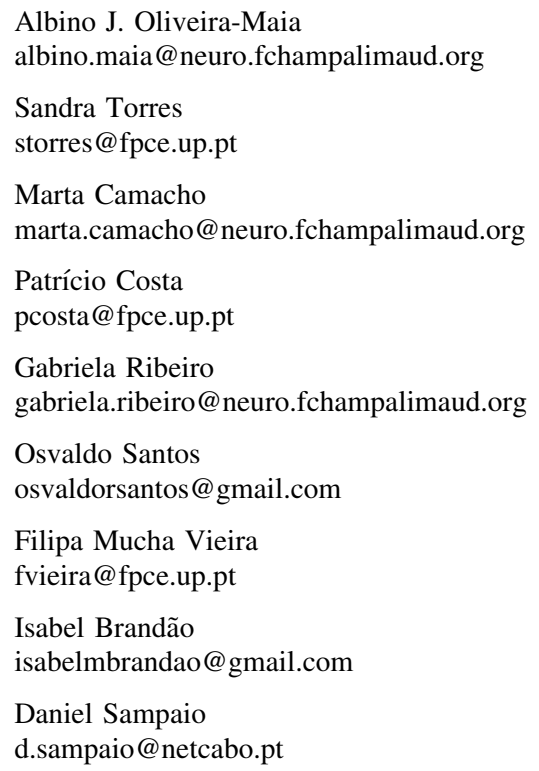

190 among obese candidates for weight-loss surgery. A battery of self-report measures of eating behavior was applied.

Results Confirmatory factor analysis verified a one-factor structure with acceptable fit, with item analysis suggesting the need to eliminate item 24 from the P-YFAS. Internal consistency $(\mathrm{KR}-20=.82)$ and test-retest stability were adequate. Correlation analyses supported convergent and divergent validity of the P-YFAS, particularly in the clinical sample. Both FA symptom count and diagnosis, according to the P-YFAS, adequately discriminated between samples, with classification of FA met by 2.5 and $25.8 \%$ of the participants in the non-clinical and clinical samples, respectively.

1 Faculdade de Psicologia e de Ciências da Educação, Universidade do Porto, Rua Alfredo Allen, 4200-135 Porto, Portugal

2 Center for Psychology, Universidade do Porto, Rua Alfredo Allen, 4200-135 Porto, Portugal

3 Champalimaud Clinical Centre, Champalimaud Centre for the Unknown, Avenida de Brasília, Doca de Pedrouços, 1400-038 Lisbon, Portugal

4 Life and Health Sciences Research Institute (ICVS), Escola de Ciências da Saúde, Universidade do Minho, Campus de Gualtar, 4710-057 Braga, Portugal

5 ICVS/3B's, PT Government Associate Laboratory, Campus de Gualtar, 4710-057 Braga, Portugal

6 Lisbon Academic Medical Centre, Faculdade de Medicina da Universidade de Lisboa, Avenida Prof. Egas Moniz, 1649-028 Lisbon, Portugal

7 Instituto de Medicina Preventiva e Saúde Pública, Faculdade de Medicina da Universidade de Lisboa, Avenida Prof. Egas Moniz, 1649-028 Lisbon, Portugal 
Conclusions These findings reinforce the use of P-YFAS in non-clinical and clinical populations. Future directions for extending YFAS validation are discussed.

Keywords Food addiction - Obesity · Reward . Psychometrics

\section{Introduction}

Obesity is a serious public health problem across the world and, despite increased efforts of prevention and treatment, its prevalence has risen substantially [2], sustaining the need for development of novel etiological hypotheses and strategies for management of this condition [3]. While the importance of exposure to calorie-rich environments is relatively consensual, the factors explaining differential susceptibility for obesity among individuals exposed to such environments are controversial [4]. Factors similar to those seen in substance dependence and addiction have been proposed to underlie the individual risk of obesity, in a process characterized by food cravings, loss of control over consumption of calorie-dense foods, increased intake over time, and unsuccessful efforts to eat less [5, 6]. Such process of "food addiction" (FA) is thought to lead to overeating, which in turn contributes to weight gain [7, 8]. Nevertheless, FA is only one among several etiological factors underlying obesity, and it is neither ubiquitous among obese patients, nor sufficient for the occurrence of obesity [9].

Evidence for validity of the FA construct, both at a behavioral and a neurobiological level, has been

8 Instituto de Saúde Ambiental, Faculdade de Medicina da Universidade de Lisboa, Avenida Prof. Egas Moniz, 1649-028 Lisbon, Portugal

9 Department of Psychiatry, Centro Hospitalar de S. João, Alameda Prof. Hernâni Monteiro, 4200-319, Porto, Portugal

10 Faculdade de Medicina da Universidade do Porto, Alameda Prof. Hernâni Monteiro, 4200-319, Porto, Portugal

11 Department of Psychiatry, Centro Hospitalar de Lisboa Norte, Avenida Prof. Egas Moniz, 1649-035 Lisbon, Portugal

12 Department of Psychiatry, Faculdade de Medicina da Universidade de Lisboa, Avenida Prof. Egas Moniz, 1649-028 Lisbon, Portugal

13 Department of Psychiatry and Mental Health, Centro Hospitalar de Lisboa Ocidental, Rua da Junqueira, 1349-019 Lisbon, Portugal

14 NOVA School of Medicine I Faculdade de Ciências Médicas, Universidade Nova de Lisboa, Campo dos Mártires da Pátria, 1169-056 Lisbon, Portugal

15 Champalimaud Research, Champalimaud Centre for the Unknown, Avenida de Brasília, Doca de Pedrouços, 1400-038 Lisbon, Portugal progressively supported by research with humans and with animal models [10]. Nevertheless, the concept remains controversial, and it is clear that further research is warranted to better define and understand the behavioral and neurobiological profile of FA [11]. This growing area of research has been supported by development of the Yale Food Addiction Scale YFAS; [12], a self-report measure of addiction-like eating behaviors, based on the diagnostic criteria for substance dependence, as defined in the DSMIV-TR [13]. This instrument allows for an examination of the psychological, behavioral, cognitive and physiological indicators of addiction-like responses to food [14]. Even considering recent changes in the criteria for substance use disorders, proposed in the DSM-V, the overlap with previous criteria supports its continued use [15].

The YFAS is currently available in many languages $[12,16-20]$, and most studies suggest that it is psychometrically sound for the study of FA. Support for validity of the YFAS has also emerged in a variety of relevant populations, namely patients with eating disorders [19] or who are overweight or obese [21], including those in weight-loss surgery programs $[22,23]$. According to a recent meta-analysis, the mean prevalence of FA as assessed by the YFAS was $11.1 \%$ in normal-weight individuals, while in patients with overweight/obesity, it increased to $24.9 \%$, and was even higher (57.6\%) among those with binge eating disorder (BED) and bulimia nervosa [24]. However, there are also data that question the relevance of FA in the context of obesity and weight-loss treatment. In fact, while Burmeister et al. [1] confirmed that elevated scores on the YFAS were related to less success after 7 weeks of weight-loss treatment, other studies have found only a trend [23] or no effect, at all, of FA on weight loss [25]. Further research is, thus, necessary to clarify the predictive validity of FA in the context of weight-loss treatments, namely through use of the YFAS. This study was aimed to validate the YFAS for use in Portuguese-speaking adult populations.

\section{Methods}

\section{Participants}

This study was conducted using two separate samples. The non-clinical sample consisted of 278 participants, recruited from three educational institutions using non-probabilistic sampling. The clinical sample, on the other hand, was composed of 190 participants with obesity, recruited using sequential sampling at weight-loss surgery clinics in three Portuguese hospitals (Centro Hospitalar de São João, Hospital do Espírito Santo, and Hospital São BernardoCentro Hospitalar de Setúbal). Participants younger than 
18 years of age were not eligible. Those with self-reported active neurological, psychiatric, gastrointestinal, hepatic or pancreatic diseases, illicit substance use or alcohol abuse and dependence, prior major gastrointestinal surgery, intragastric balloon or history of food allergies, as well as those who were pregnant, breastfeeding, illiterate or did not understand instructions for study, were excluded.

\section{Material}

Yale Food Addiction Scale (YFAS). The YFAS [12] consists of 25 self-report items measuring addiction-like eating behaviors of high fat/sugar foods, occurring over the prior year. The seven symptoms of substance dependence, as outlined in the DSM-IV-TR (American Psychiatric Association, 2000), were revised to apply to eating behavior: consumption in greater quantity and for longer than intended; desire or unsuccessful effort to cut down or quit consumption; large amount of time and effort to obtain, consume, and recover from consumption; important social, occupational, or recreational activities given up or reduced; continued behavior despite knowledge of adverse physical and psychological consequences; tolerance; and withdrawal. In addition to 20 items distributed across the seven criteria (symptoms), 2 items are designed to assess clinically significant impairment, and 3 items serve as primers for other questions. Each symptom is satisfied when one or more of the respective items are endorsed. The YFAS provides two scoring options, a continuous version of the scale (YFAS symptom count) that indicates the number of dependence symptoms that have been met (scores range from 0 to 7), and a dichotomous version that provides a diagnosis of FA (i.e., FA status yes/no) when the respondent presents at least three symptoms and reports clinically significant impairment and/or distress.

Other self-report instruments. The Portuguese Power of Food Scale P-PFS [26] is a Portuguese version of the PFS [27], a 15-item questionnaire that assesses psychological impact of an environment with high availability of palatable foods. It rates three dimensions of proximity to food: food available but not physically present, food physically present but not yet tasted, and food tasted but not yet consumed. For this study, Cronbach's alpha $(\alpha)$ of the P-PFS was .88 for the non-clinical sample and .93 for the clinical sample. The Portuguese Dutch Eating Behavior Questionnaire P-DEBQ [28] is a Portuguese version of the DEBQ [29], a 33-item questionnaire assessing different aspects of eating behaviors, distributed in three subscales: restrained eating, emotional eating and external eating (non-clinical sample $-\alpha=.93$; clinical sample $-\alpha=.90$ ). The Portuguese Eating Disorder Inventory P-EDI [30] is a Portuguese version of the EDI [31], a 64-item measure designed to assess psychological and behavioral traits associated with eating disorders, across eight subscales: drive for thinness, bulimia, body dissatisfaction, ineffectiveness, perfectionism, interpersonal distrust, interoceptive awareness and maturity fears (non-clinical sample$\alpha=.88$ ). The Portuguese Binge Eating Scale P-BES [32] is a Portuguese version of the BES [33], a 16-item scale to assess severity of binge eating using behavioral, cognitive and affective symptoms (non-clinical sample- $\alpha=.89$ ). The Newest Vital Sign (NVS) [34] is a screening test designed to assess health literacy. A Portuguese version (PNVS) is currently being developed [35] (non-clinical sample $-\alpha=.78$; clinical sample $-\alpha=.89$ ).

Structured Clinical Interview for DSM-IV Axis I Disorders (SCID-I). The SCID-I [36], a semi-structured clinical interview for diagnosis of psychiatric disorders according to DSM-IV Axis I, translated and adapted to Portuguese by Tavares [37], was used to assess lifetime and current BED diagnosis in the clinical sample. Different raters applied this interview in the North and South of Portugal, allowing for assessment of the association between rater and SCID/BED diagnosis. The Chi-squared test for independence (with Yates' continuity correction) indicated that there was no significant association between these variables $\left(\chi^{2} \quad(1)=.81, p=.276\right.$, $\varphi=.10)$.

Body mass index (BMI). The BMI $\left(\mathrm{kg} / \mathrm{m}^{2}\right)$ of the nonclinical participants was calculated according to self-reported height and weight values provided by participants. In the clinical sample, height and weight were obtained by direct measurement with a SECA digital scale and stadiometer.

\section{Procedures}

Permission for translation and validation of a Portuguese version of the YFAS was granted by the original authors (Ashley Gearhardt). The translation process was based on the back-translation technique [38]. The study protocol was approved by Ethics Committees at the Champalimaud Foundation, Faculdade de Medicina da Universidade de Lisboa, Universidade de Évora, Centro Hospitalar de São João and Hospital São Bernardo-Centro Hospitalar de Setúbal. Written informed consent was obtained from all subjects.

A pilot study was carried out with a preliminary version of the Portuguese YFAS (P-YFAS), administered to 7 individuals with obesity and 10 normal-weight individuals, of both genders. Pilot participants were interviewed to assess their reactions to item difficulty, wording and meaning, as well as to the instructions for administration, leading to minor adaptations on wording of several items and a final version of the P-YFAS. 
In the non-clinical and clinical samples, self-administered questionnaires were applied using a paper-and-pencil format, completed after socio-demographic information (gender, age, and education) and anthropometric data were collected. The assessment protocol differed slightly between samples: YFAS, PFS, DEBQ and NVS were applied to both samples, the EDI and BES only to the nonclinical sample, and the SCID-I (BED diagnosis) only to the clinical sample. To assess test-retest reliability, the YFAS was re-administered 4 weeks later in a subgroup of 104 participants of the non-clinical sample. This interval was chosen to allow for comparisons with existing data [18]. Temporal stability of the scale was also verified in 30 participants of the clinical sample, with time intervals of up to 8 months, which are closer to customary clinical reassessment time points.

\section{Data analysis}

Data analysis was focused on exploring the psychometric properties of the P-YFAS. Based on the one-factor model found in previous studies e.g., [12, 17, 20, 22], a confirmatory factor analysis for dichotomous data (not including the primer items) was conducted on Mplus version 6.0, using a mean- and variance-adjusted weighted least square (WLSMV) estimator with a polychoric correlation matrix. To assess model fit, several criteria were considered: root mean square error of approximation (RMSEA) <.06 [39], comparative fit index $(\mathrm{CFI})>.90$ [40], weighted root mean square residual $(\mathrm{WRMR})<1.0$ [41], non-significant Chisquared $\left(\chi^{2}\right)$ test $(p<.05)$ [42], and normed Chi-squared $\left(\chi^{2} / d f\right)<3.0$ [43]. Internal reliability was assessed using the Kuder-Richardson's alpha (KR-20) and, in the non-clinical sample, test-retest reliability was estimated using the twoway mixed, single-measure intraclass correlation coefficient (ICC). To test temporal stability of P-YFAS symptom count in the clinical sample, two subsamples were considered according to test-retest time intervals, namely short (6-8 weeks) and long (3-8 months) intervals. Pairedsamples $t$ tests were conducted to analyze whether there were differences in the means of these subsamples at the two time points.

Distributions of continuous measures (total scores and subscales) used to analyze YFAS validity were examined for normality, independently in clinical and non-clinical samples, and found to be normally distributed, as per analysis of kurtosis, skewness and comparison of mean and median. Association between P-YFAS diagnosis and age, education (years of schooling) and BMI was examined using two-tailed Student's $t$ tests (with Cohen's $d$ to provide a measure of effect size), and association with gender using independence $\chi^{2}$ tests (with Yates' continuity correction). Convergent validity was assessed using Pearson correlation coefficients $(r)$ between P-YFAS symptom count and other instruments measuring eating behaviors, specifically instruments most linked to binge eating and/or reward-related eating. The P-PFS and selected P-DEBQ subscales (emotional eating and external eating) were used in both samples, while the P-BES total score and selected P-EDI subscales (bulimia, body dissatisfaction, and drive for thinness, representing the best screen for BED according to the recent literature [44]), were used in the non-clinical sample. Divergent validity was assessed using Pearson correlations between P-YFAS symptom count and related but distinct constructs, following the same procedure adopted in previous psychometric studies on the YFAS. Here, we considered the dietary restraint (DEBQ restrained eating subscale) and health literacy (NVS) constructs. In the non-clinical sample, three P-EDI subscales (maturity fears, perfectionism, and interpersonal distrust, chosen based on their low screening properties for BED [44]) were additionally used to test divergent validity. To assess discriminative validity, we performed a one-way analysis of covariance (ANCOVA) to compare the mean scores of the P-YFAS symptom count in clinical and nonclinical samples, while adjusting for age and education.

With the exception noted above, data analyses were performed using SPSS version 22.0. All analyses were twotailed, with significance considered at $p<.05$.

\section{Results}

In the non-clinical and clinical samples, participation rate was 97.8 and $88.8 \%$, respectively. See Table 1 for a summary of socio-demographic and clinical characteristics for participants of both samples.

\section{P-YFAS factor structure and reliability}

Confirmatory factor analysis with P-YFAS dichotomous items achieved satisfactory goodness of fit for a one-dimensional solution (data not shown). Internal reliability was good $(\mathrm{KR}-20=.80)$ and, with one exception, all items presented a significant coefficient. Item \#24 presented a non-significant coefficient and a very low item-total correlation (.06), prompting its exclusion and repetition of analyses (see supplementary material, Table S1). After excluding item \#24, fit statistics improved $\left[\chi^{2}(189)=456\right.$, $p<.001, \chi^{2} / d f=2.41 ;$ RMSEA $=.06,90 \%$ CI $.05 / .06, \mathrm{p}$ $(\mathrm{RMSEA}<.05)=.06 ; \mathrm{CFI}=.90 ; \mathrm{WRMR}=1.41]$ with satisfactory fit achieved for all indices with the exception of the $\chi^{2}$ test. The latter was significant possibly due to the fact that the $\chi^{2}$ statistic is sensitive to sample size. The KR20 internal reliability coefficient was .82 and all items loaded significantly on the common factor $(p<.001)$, with 
Table 1 Socio-demographic and clinical information of the non-clinical and the clinical samples

\begin{tabular}{lllllr}
\hline & & Non-clinical $(n=278)$ & Clinical $(n=190)$ & $t$ & \multicolumn{1}{c}{$d$} \\
\hline Age & $M(\mathrm{SD})$ & $23.06(6.43)$ & $43.21(10.39)$ & $-23.80^{* * * *}$ & -2.33 \\
Education & $M(\mathrm{SD})$ & $11.31(2.13)$ & $9.72(4.26)$ & $4.76^{* * *}$ & .47 \\
BMI & $M(\mathrm{SD})$ & $22.65(3.35)$ & $43.24(5.80)$ & $-44.04 * * *$ & -4.35 \\
Gender & $(\%$ women $)$ & $66.5 \%$ & $87.4 \%$ & $\chi^{2}=25.00^{* * *}$ & \\
\hline
\end{tabular}

$B M I$ body mass index, Education years of formal education $* * * p<.001$ item-total correlations between .20 and .60. Five items (\#4, $\# 11$, \#21, \#22, and \#25) had low item-total correlations (.20 to .30), but KR-20 did not decrease when these items were deleted (see supplementary material, Table S1). Thus, a one-factor model without item \#24 was the factorial structure proposed for the P-YFAS. To verify differences between the P-YFAS and the original YFAS, all the following analyses were performed with both versions. Since differences between the two versions were not significant, only the results regarding the final 21-item P-YFAS are presented from here on.

Test-retest reliability in the non-clinical sample was adequate (ICC $=.64,95 \%$ CI .51-.74). In the clinical sample, there were no significant differences in the YFAS mean scores for test and retest, after both short $\left(t_{(12)}=-0.39, p=.701, \eta^{2}=.01\right)$ and long $\left(t_{(14)}=0.61\right.$, $\left.p=.551, \eta^{2}=.03\right)$ reassessment intervals.

\section{FA diagnosis: prevalence and associated socio- demographic and clinical features}

FA diagnosis was ascertained using the P-YFAS in all subjects from the non-clinical sample, and in all but 12 subjects from the clinical sample. For those 12 subjects, this was not possible due to missing data. FA was, thus, diagnosed in $2.5 \%(n=7)$ of the non-clinical sample and $25.8 \%(n=46)$ of the clinical sample. Similar FA rates were found using the 22-item version of the P-YFAS (2.9 and $25.8 \%$, respectively). Endorsement rates of each symptom assessed by the P-YFAS are available as supplementary material (Table S2). Associations between FA diagnosis and gender were not significant in the non-clinical $\left(\chi_{(1, n=278)}^{2}=.02, p=.898, \varphi=-.03\right)$ or the clinical sample $\left(\chi_{(1, n=178)}^{2}=.42, p=.515, \varphi=-.07\right)$. FA diagnosis was also not associated with years of schooling and BMI (Table 2). Differences in FA diagnosis were found only according to age in the non-clinical sample, where individuals with FA diagnosis were found to be younger $(M=20.29, \mathrm{SD}=1.98)$ than the remaining sample $\quad\left(M=23.13, \quad \mathrm{SD}=6.31 ; \quad t_{\mathrm{Welch}(9.8)}=3.37\right.$, $p=.007, d=.59)$. Given the small number of individuals with FA diagnosis in the non-clinical sample, this finding should be considered with caution.

\section{Convergent, divergent, and discriminative validity}

Table 3 shows correlations between P-YFAS symptom count and clinical measures selected to test convergent and divergent validity. When compared with other measures relevant to eating behavior, the P-YFAS achieved adequate convergent validity in the non-clinical sample $(.216<r<.487, p<.001)$, and good convergent validity $(.412<r<.727, \quad p<.001)$ in the clinical sample. Regarding divergent validity, correlations were non-significant, with exception of the DEBQ restrained eating subscale, which correlated slightly, but significantly, with P-YFAS symptom count in both samples.

Discriminative validity of the scale was supported by finding of a significant difference between the two samples on P-YFAS symptom count scores (non-clinical sample: $M=1.55, \quad \mathrm{SD}=1.31 ; \quad$ clinical sample: $\quad M=2.81$, $\mathrm{SD}=1.87)$, even after adjusting for age and education $\left(F_{(1,452)}=25.25, p<.001, \eta_{p}^{2}=.05\right.$; ANCOVA $)$. Among the clinical sample, the proportion of individuals with an FA diagnosis $(25.8 \%)$ was also significantly higher than what was found for the non-clinical sample (2.5\%; $\chi_{(1, n=456)}^{2}=55.23, p<.001, \varphi=.36 ; \chi^{2}$ test $)$.

\section{Discussion}

This study describes the translation of the YFAS [12] into Portuguese (P-YFAS), with assessment of its psychometric properties, in terms of validity (construct, convergent, divergent, and discriminative) and reliability (internal consistency and test-retest), measured in two adult samples (non-clinical and clinical). We confirmed the previously proposed one-factor structure of the YFAS after excluding item \#24 (unsuccessful effort to cut down), given its nonsignificant coefficient and very low item-total correlation. Exclusion of item \#24 has been reported in other versions of the scale [16-20,22], with suggestions towards its removal from the questionnaire $[16,17,20]$. Both in our study and those mentioned above, item \# 24, along with others that compose the criterion for "persistent desire or unsuccessful attempts to quit" (items \#4, \#22, \#25), allow only for a slight differentiation between food-addicted and 
Table 2 Differences in age, education, and BMI according to P-YFAS diagnostic threshold

Table 3 Convergent and divergent validity

\begin{tabular}{|c|c|c|c|c|c|}
\hline & $\begin{array}{l}\text { No food addiction } \\
M \text { (SD) }\end{array}$ & $\begin{array}{l}\text { Food addiction } \\
M(\mathrm{SD})\end{array}$ & $t$ & $p^{*}$ & Cohen's $d$ \\
\hline \multicolumn{6}{|c|}{ Non-clinical sample $(n=278)$} \\
\hline Age & $23.13(6.31)$ & $20.29(1.98)$ & 3.37 & .007 & .59 \\
\hline Education & $11.32(2.15)$ & $10.57(1.13)$ & 1.68 & .136 & .44 \\
\hline BMI & $22.61(3.35)$ & $24.11(3.24)$ & -1.17 & .245 & -.45 \\
\hline \multicolumn{6}{|c|}{ Clinical sample $(n=178)$} \\
\hline Age & $42.49(10.04)$ & $44.91(11.26)$ & -1.36 & .174 & -.23 \\
\hline Education & $10.07(4.07)$ & $9.50(4.81)$ & .72 & .476 & .13 \\
\hline BMI & $43.10(5.47)$ & $44.33(6.86)$ & -1.22 & .224 & -.20 \\
\hline
\end{tabular}

$B M I$ body mass index; Education years of formal education

* Significant $p$ values are in bold

\begin{tabular}{|c|c|c|}
\hline & \multicolumn{2}{|c|}{ P-YFAS symptom count } \\
\hline & Non-clinical sample & Clinical sample \\
\hline \multicolumn{3}{|l|}{ Convergent validity } \\
\hline P-PFS-total score & $.413 * * *$ & $.727 * * *$ \\
\hline P-PFS—food available & $.449 * * *$ & $.701 * * *$ \\
\hline P-PFS—food present & $.216^{* * *}$ & $.610 * * *$ \\
\hline P-PFS—food tasted & $.310 * * *$ & $.615^{* * *}$ \\
\hline P-DEBQ - external eating & $.449 * * *$ & $.608 * * *$ \\
\hline P-DEBQ — emotional eating & $.346^{* * * *}$ & $.624 * * *$ \\
\hline P-EDI—bulimia & $.317 * * *$ & - \\
\hline P-EDI—body dissatisfaction & $.319 * * *$ & - \\
\hline P-EDI-drive for thinness & $.303 * * *$ & - \\
\hline Binge eating $^{\mathrm{a}}$ & $.487 * * *$ & $.412 * * *$ \\
\hline \multicolumn{3}{|l|}{ Divergent validity } \\
\hline P-NVS & -.030 & -.071 \\
\hline P-DEBQ—restrained eating & $.236 * *$ & $-.165^{*}$ \\
\hline P-EDI—maturity fears & .101 & - \\
\hline P-EDI-perfectionism & .139 & - \\
\hline P-EDI-interpersonal distrust & .128 & - \\
\hline
\end{tabular}

- indicates that the measure was only available for non-clinical sample

$P$-YFAS Portuguese version of the Yale Food Addiction Scale, P-PFS Portuguese version of the Power of Food Scale, $P-D E Q B$ Portuguese version of the Dutch Eating Behavior Questionnaire, $P$-EDI Portuguese version of the Eating Disorder Inventory, $P-N V S$ Portuguese version of the Newest Vital Sign

$* p<.05 ; * * p<.01 ; * * * p<.001$

${ }^{a}$ Binge eating was assessed by the Binge Eating Scale (P-BES), in non-clinical sample, and the Structured Clinical Interview for DSM-IV Axis I Disorders (SCID-I), in clinical sample non-addicted individuals (see Table S2). We believe that the low discriminative power of these items could result from the prevalence of dieting in the general population [45]. Important next steps in FA research include further refinement of the YFAS, with selection of the more specific items associated to FA.

The P-YFAS exhibited good internal reliability, and test-retest analysis also supported stability of the scale. As yet, only two studies have investigated the stability of the YFAS across time $[18,46]$. Both were conducted with non-clinical populations and achieved an ICC slightly higher than what was found here, also for the non-clinical population $(\sim .70)$. Here, we also report, to our knowledge for the first time, preliminary data for temporal stability of the YFAS in a clinical population. In this population, even though we tested a smaller group of individuals $(n=30)$ over longer time intervals (up to 8 months), the P-YFAS was found to be relatively stable at both shorter (6-8 weeks) and longer (3-8 months) intervals. 
Using the P-YFAS, we found an FA prevalence of $2.5 \%$ in the non-clinical sample and $25.8 \%$ in the clinical sample. To date, three studies analyzed the prevalence of FA in weight-loss surgery candidates and reported considerably higher rates: $31.8 \%$ [47], 41.7\% [22], and 53.7\% [23]. Comparing the samples of these studies, we did not find striking differences regarding age, and there are insufficient data to allow for comparisons of BMI. The FA rate in our non-clinical sample was also lower than in previous studies using student [16, 18] and population-based samples [17], with prevalence rates around 9\%. Only one study [48] found a rate similar to ours $(1.6 \%)$, but in participants classified as underweight or normal-weight. In our sample, these BMI classes represented $80.2 \%$ of the participants, which may have had some impact in FA assessment. However, given the limited evidence for differences in FA prevalence according to weight or age categories [9], this hypothesis should be considered cautiously, and the impact of these and other clinical and socio-demographic variables on the expression of FA symptoms should be further explored. In interpretation of our findings, we are more inclined to argue that the FA assessment can be sensitive to cultural eating habits and food preferences. Culture can affect attitudes towards food, differences in accepted portion sizes, and personal feelings of embarrassment and distress towards overeating [49]. It is possible that in Portugal, a country with rich gastronomic tradition, people view larger food portions as more acceptable than in other countries. The question regarding cultural differences in FA symptomatology is, thus, in our view, essential to extend validation of the YFAS.

The P-YFAS symptom count score was shown to have adequate convergent and divergent validity, with moderate to high correlations between the P-YFAS score and measures of eating pathology. Contrary to our expectation, in divergent validity analysis, we observed small yet significant correlations between P-YFAS and P-DEBQ restrained eating subscale scores. In fact, these two constructs, apparently with opposite expressions (restrictive eating vs. overeating), can be related to the extent that intense dieting may result in persistent hunger and limit cognitive control, making dieters susceptible to disinhibited eating. Dietary restraint may, thus, represent an "intent to diet" rather than caloric restriction per se [29]. Discriminative validity of the P-YFAS was also supported given that, after adjusting for age and education, the symptom count score was able to differentiate between clinical and non-clinical samples, and the P-YFAS diagnostic also differed significantly according to sample group (clinical vs. non-clinical).

The results of our study must be interpreted in the context of the study design. The main findings are based on self-reported measures, implying risks regarding accuracy [50]. Generalizability of our findings is also a concern, with the clinical sample predominantly female and comprising a specific group of patients with obesity: candidates for weight-loss surgery. Confirmation of the psychometric properties of the P-YFAS should, thus, be performed with larger samples of individuals, including broader profiles of patients with obesity. Specifically with regards to shortand mid-term stability of the scale, future research should extend current findings in both samples, since the mid-term stability in non-clinical samples has been analyzed only once [46], and the data we provide regarding stability in clinical samples are preliminary due to the small number of participants that were re-assessed. Finally, the unbalance in sample sizes in this study, resulting from the smaller size of our clinical sample, limited the testing of whether the same P-YFAS configuration holds across groups. In future studies, it would be pertinent to test measurement invariance between groups, namely between clinical and nonclinical samples.

\section{Conclusions}

Here, we have translated and adapted the Portuguese version of the YFAS in non-clinical and clinical samples, and found it to have adequate psychometric properties. Nevertheless, our data raised questions related to conceptual definition of the symptom "unsuccessful effort to cut down", which seems to be a broad and unspecific symptom. Furthermore, the lower percentage of FA diagnosis found in our samples suggests that the YFAS is sensitive to cultural food habits and preferences. Future directions for extending YFAS validation should include the clarification of the impairments specific to FA condition and their relationship with clinical and socio-demographic variables, distinctively in clinical and nonclinical samples.

Acknowledgements The authors would like to thank Ashley Gearhardt (Department of Psychology, University of Michigan) for providing access to the Yale Food Addiction Scale and contributing to the adaptation process. We would also like to thank Centro Hospitalar de S. João, Hospital São Bernardo, Faculdade de Ciências da Nutrição e Alimentação da Universidade do Porto, Hospital Espírito Santo de Évora, Instituto do Emprego e Formação Profissional, Universidade Atlântica and Universidade de Évora, in particular Ana André, André Ferreira, Andreia Santos, António-Roma-Torres, Armando Raimundo, Carlos Trindade, Cecília Silva, Cristina Pontes, Manuel Carvalho, Margarida Pegacho, Mónica Silva, Rita Fernandes and Sara Pacheco, for assistance with recruitment of participants.

\section{Compliance with ethical standards}

Funding This work was supported by Fundação para a Ciência e Tecnologia through a Junior Research and Career Development Award from the Harvard Medical Portugal Program (HMSP/ICJ/ 0020/2011) to AJO-M and CPUP (UID/PSI/00050/2013) to ST, and a Grant from the BIAL Foundation (176/10) to AJO-M. 
Conflict of interest On behalf of all authors, the corresponding author states that there is no conflict of interest.

Ethical approval All procedures performed in this study were in accordance with the ethical standards of the institutional and/or national research committee and with the 1964 Helsinki declaration and its later amendments or comparable ethical standards.

Informed consent Informed consent was obtained from all individual participants included in the study.

\section{References}

1. Burmeister JM, Hinman N, Koball A, Hoffmann DA, Carels RA (2013) Food addiction in adults seeking weight loss treatment. Implications for psychosocial health and weight loss. Appetite 60:103-110. doi:10.1016/j.appet.2012.09.013

2. Ng M, Fleming T, Robinson M, Thomson B, Graetz N, Margono $\mathrm{C}$ et al (2014) Global, regional, and national prevalence of overweight and obesity in children and adults during 1980-2013: a systematic analysis for the Global Burden of Disease Study 2013. Lancet 384:766-781. doi:10.1016/S0140-6736(14)60460-8

3. Oliveira-Maia J, Roberts CD, Simon SA, Nicolelis MAL (2011) Gustatory and reward brain circuits in the control of food intake. Adv Tech Stand Neurosurg 36:31-59. doi:10.1007/978-3-70910179-7_3

4. Schulte E, Joyner M, Potenza M, Grilo C, Gearhardt A (2015) Current considerations regarding food addiction. Curr Psychiatry Rep 17:1-8. doi:10.1007/s11920-015-0563-3

5. Fortuna JL (2012) The obesity epidemic and food addiction: clinical similarities to drug dependence. J Psychoact Drugs 44:56-63. doi:10.1080/02791072.2012.662092

6. Gearhardt AN, Corbin WR, Brownell KD (2009) Food addiction: an examination of the diagnostic criteria for dependence. J Addict Med 3:1-7. doi:10.1097/ADM.0b013e318193c993

7. Gearhardt AN, White MA, Potenza MN (2011) Binge eating disorder and food addiction. Curr Drug Abus Rev 4:201-207

8. Davis C, Carter JC (2009) Compulsive overeating as an addiction disorder. A review of theory and evidence. Appetite 53:1-8. doi:10.1016/j.appet.2009.05.018

9. Meule A (2011) How prevalent is 'food addiction'? Front Psychiatry 2:1-4. doi:10.3389/fpsyt.2011.00061

10. Davis C (2013) From passive overeating to "Food Addiction": a spectrum of compulsion and severity. ISRN Obes 2013:1-20. doi:10.1155/2013/435027

11. Ziauddeen H, Fletcher PC (2013) Is food addiction a valid and useful concept? Obes Rev 14:19-28. doi:10.1111/j.1467-789X. 2012.01046.x

12. Gearhardt AN, Corbin WR, Brownell KD (2009) Preliminary validation of the Yale Food Addiction Scale. Appetite 52:430-436. doi:10.1016/j.appet.2008.12.003

13. American Psychiatric Association (2000) Diagnostic and statistical manual of mental disorders, 4th edn. American Psychiatric Press, Washington

14. Meule A, Gearhardt AN (2014) Five years of the Yale Food Addiction Scale: Taking stock and moving forward. Curr Addict Rep 1:193-205. doi:10.1007/s40429-014-0021-z

15. Meule A, Gearhardt AN (2014) Food Addiction in the Light of DSM-5. Nutrients 6:3653-3671. doi:10.3390/nu6093653

16. Meule A, Vögele C, Kübler A (2012) German translation and validation of the Yale Food Addiction Scale. Diagnostica 58:115-126. doi:10.1026/0012-1924/a000047
17. Brunault P, Ballon N, Gaillard P, Réveillère C, Courtois R (2014) Validation of the French version of the Yale Food Addiction Scale: an examination of its factor structure, reliability, and construct validity in a nonclinical sample. Can J Psychiatry 59:276-284

18. Chen G, Tang Z, Guo G, Liu X, Xiao S (2015) The Chinese version of the Yale Food Addiction Scale: an examination of its validation in a sample of female adolescents. Eat Behav 18:97-102. doi:10.1016/j.eatbeh.2015.05.002

19. Granero R, Hilker I, Agüera Z, Jiménez-Murcia S, Sauchelli S, Islam MA et al (2014) Food Addiction in a Spanish Sample of Eating Disorders: DSM-5 Diagnostic Subtype Differentiation and Validation Data. Eur Eat Disord Rev 22:389-396. doi:10.1002/ erv. 2311

20. Innamorati M, Imperatori C, Manzoni G, Lamis D, Castelnuovo G, Tamburello A et al (2015) Psychometric properties of the Italian Yale Food Addiction Scale in overweight and obese patients. Eat Weight Disord 20:119-127. doi:10.1007/s40519014-0142-3

21. Davis C, Curtis C, Levitan RD, Carter JC, Kaplan AS, Kennedy JL (2011) Evidence that food addiction is a valid phenotype of obesity. Appetite 57:711-717. doi:10.1016/j.appet.2011.08.017

22. Meule A, Heckel D, Kübler A (2012) Factor structure and item analysis of the Yale Food Addiction Scale in obese candidates for bariatric surgery. Eur Eat Disord Rev 20:419-422. doi:10.1002/ erv. 2189

23. Clark SM, Saules KK (2013) Validation of the Yale Food Addiction Scale among a weight-loss surgery population. Eat Behav 14:216-219. doi:10.1016/j.eatbeh.2013.01.002

24. Pursey KM, Stanwell P, Gearhardt AN, Collins CE, Burrows TL (2014) The prevalence of food addiction as assessed by the Yale Food Addiction Scale: a systematic review. Nutrients 6:4552-4590. doi:10.3390/nu6104552

25. Lent MR, Eichen DM, Goldbacher E, Wadden TA, Foster GD (2014) Relationship of food addiction to weight loss and attrition during obesity treatment. Obesity 22:52-55. doi:10.1002/oby.20512

26. Ribeiro G, Santos O, Camacho M, Torres S, Mucha-Vieira F, Sampaio D et al (2015) Translation, cultural adaptation and validation of the power of food scale for use by adult populations in Portugal. Acta Med Port 28:575-582

27. Lowe MR, Butryn ML, Didie ER, Annunziato RA, Thomas JG, Crerand CE et al (2009) The Power of Food Scale. A new measure of the psychological influence of the food environment. Appetite 53:114-118. doi:10.1016/j.appet.2009.05.016

28. Viana V, Sinde S (2003) Estilo Alimentar: Adaptação e validação do Questionário Holandês do Comportamento Alimentar. Psic Teor Inve Prat 1:59-71

29. Van Strien T, Frijters JER, Bergers G, Defares PB (1986) The Dutch Eating Behavior Questionnaire (DEBQ) for assessment of restrained, emotional, and external eating behavior. Int $\mathrm{J}$ Eat Disord 5:295-315

30. Machado PP, Gonçalves S, Martins C, Soares IC (2001) The Portuguese version of the Eating Disorders Inventory: Evaluation of its psychometric properties. Eur Eat Disord Rev 9:43-52. doi:10.1111/papt.12048

31. Garner DM, Olmsted MP, Polivy J (1983) Development and validation of a multidimensional Eating Disorder Inventory for anorexia nervosa and bulimia. Int J Eat Disord 2:15-34

32. Tapadinhas AR, Pais-Ribeiro J (2012) Validação da escala de ingestão compulsiva (BES). Um estudo com uma amostra de obesos portugueses. Atas do $9^{\circ}$ Congresso Nacional de Psicologia da Saúde. Lisboa: Placebo Editora. pp 1330-35

33. Gormally J, Black S, Daston S, Rardin D (1982) The assessment of binge eating severity among obese persons. Addict Behav 7:47-55. doi:10.1016/0306-4603(82)90024-7 
34. Weiss BD, Mays MZ, Martz W, Castro KM, DeWalt DA, Pignone MP et al (2005) Quick assessment of literacy in primary care: the newest vital sign. Ann Fam Med 3:514-522. doi:10. 1370/afm.405

35. Santos O, Oliveira A, Carvalho C, Lunet N, Azevedo A, Paiva D et al (2012) Self-administration of the Newest Vital Sign in Portuguese young adults: study of its internal reliability. Int $\mathbf{J}$ Behav Med 19:S189

36. Spitzer RL, Williams JBW, Gibbon M, First MB (1990) Structured Clinican Interview for DSM-III-R (SCID). American Psychiatric Press, Washington

37. Tavares M (1996) Entrevista clínica estruturada para o DSM-IV: Transtornos do eixo I-edição para pacientes (SCID-I/P 2.0). Instituto de Psicologia, Universidade de Brasília

38. Gudmundsson E (2009) Guidelines for translating and adapting psychological instruments. Nord Psychol 61:29-45. doi:10.1027/ 1901-2276.61.2.29

39. Hu L, Bentler PM (1999) Cutoff criteria for fit indexes in covariance structure analysis: Conventional criteria versus new alternatives. Struct Equ Model 6:1-55. doi:10.1080/ 10705519909540118

40. Byrne BM (2010) Structural equation modelling with AMOS: Basic concepts, applications, and programming, 2nd edn. Routledge, New York

41. Yu CY (2002) Evaluating cutoff criteria of model fit indices for latent variable models with binary and continuous outcomes. University of California, Los Angeles

42. Barrett P (2007) Structural equation modelling: Adjudging model fit. Pers Individ Differ 42:815-824. doi:10.1016/j.paid.2006.09. 018
43. Kline RB (2005) Principles and practice of structural equation modeling, 2nd edn. Guilford Press, New York

44. Mustelin L, Kärkkäinen U, Kaprio J, Keski-Rahkonen A (2016) The Eating Disorder Inventory in the screening for DSM-5 binge eating disorder. Eat Behav 22:145-148. doi:10.1016/j.eatbeh. 2016.06.011

45. de Ridder D, Adriaanse M, Evers C, Verhoeven A (2014) Who diets? Most people and especially when they worry about food. Appetite 80:103-108. doi:10.1016/j.appet.2014.05.011

46. Pursey KM, Collins CE, Stanwell P, Burrows TL (2016) The stability of 'food addiction' as assessed by the Yale Food Addiction Scale in a non-clinical population over 18-months. Appetite 96:533-538. doi:10.1016/j.appet.2015.10.015

47. Pepino MY, Stein RI, Eagon JC, Klein S (2014) Bariatric surgery-induced weight loss causes remission of food addiction in extreme obesity. Obesity 22:1792-1798. doi:10.1002/oby.20797

48. Pedram P, Wadden D, Amini P, Gulliver W, Randell E, Cahill F et al (2013) Food addiction: its prevalence and significant association with obesity in the general population. PLoS One 8:e74832. doi:10.1371/journal.pone.0074832

49. Bennett S, Dodge T (2007) Ethnic-racial differences in feelings of embarrassment associated with binge eating and fear of losing control. Int J Eat Disord 40:454-459. doi:10.1002/eat.20374

50. Vitousek KB, Daly J, Heiser C (1991) Reconstructing the internal world of the eating-disordered individual: Overcoming distortion in self-report. Int J Eat Disord 10:647-666. doi:10.1002/1098108X(199111)10:6<647:AID-EAT2260100604>3.0.CO;2-T 